\title{
Efficacy and safety of nimotuzumab in unresectable, recurrent, and/or metastatic squamous cell carcinoma of the head and neck: A hospital-based retrospective evidence
}

\author{
Sundaram Subramanian, Nithya Sridharan,V. Balasundaram', Sameer Chaudhari²
}

\begin{abstract}
Context: Role of nimotuzumab in locally advanced head and neck cancer (HNC) is well established in India; however, no clinical evidence is available for its role in recurrent and/or metastatic HNC. Aims: The aim of this study is to evaluate the efficacy and safety of nimotuzumab when added to standard treatment in unresectable, recurrent, and metastatic squamous cell carcinoma of the head and neck (SCCHN) Settings and Design: Hospital records of 14 patients diagnosed with recurrent and/or metastatic HNC with histologically confirmed squamous cell carcinoma and being treated with nimotuzumab along with standard treatments from December 2010 to December 2016 were retrospectively evaluated. Subjects and Methods: The tumor response rate and overall survival (OS) were analyzed. Toxicity and adverse events (AEs) were assessed as per common terminology criteria for adverse events $(C T C A E) \vee 4$. Results: Oral cavity was most commonly involved region followed by hypopharynx and oropharynx. At 24 weeks after completion of treatment, overall response rate (complete response (CR) + partial response (PR)) was $75 \%$. Survival rate at I, 2, and 3 years was $77.80 \%, 64.81 \%$, and $64.81 \%$, respectively. At a median follow-up of 15.17 months, median OS was not reached. All AEs were either Grade I (66.7\%) or Grade II (33.3\%). No Grade III or Grade IV AEs were observed. No added toxicity was observed due to nimotuzumab. Conclusions: In the first of its kind study, the addition of nimotuzumab to standard treatment showed promising response rate as well as survival outcomes in recurrent and/or metastatic SCCHN patients without producing additional toxicity.
\end{abstract}

Key words: Antibodies, carcinoma, head and neck neoplasms, inoperable, metastatic, monoclonal, nimotuzumab, overall survival, recurrent, squamous cell, therapy, tumor response

\section{Introduction}

Squamous cell carcinoma of the head and neck (SCCHN) is the sixth leading cancer by incidence worldwide. ${ }^{[1]}$ Cigarette smoking and alcohol are the major causative factors for $\mathrm{SCCHN}$ in the Western population, ${ }^{[2]}$ whereas the use of smokeless tobacco, betel nut, and Epstein-Barr virus are common factors responsible in the Asian population. ${ }^{[3-5]}$

Most of the cases of SCCHN present in locally advanced stage and often tend to recur either locally or develop metastatic disease despite receiving standard treatment. ${ }^{[4,6,7]}$ Patients with recurrent and/or metastatic (R/M) SCCHN have a poor prognosis and outcome. Palliative systemic chemotherapy with or without radiation therapy remains the mainstay of treatment. Platinum-based chemotherapy/5-fluorouracil (5-FU)/taxane-based regimens are the preferred standard treatment in the management of in unresectable and recurrent/metastatic SCCHN..$^{[4,8-10]}$ Despite advances in systemic chemotherapy regimens, the overall survival (OS) rate is low in recurrent/metastatic SCCHN. ${ }^{[10]}$

Epidermal growth factor receptor (EGFR) is highly expressed in more than $90 \%$ of all cases of SCCHN, and this expression is correlated with poor prognosis, including decreased survival, resistance to radiotherapy (RT), locoregional treatment failure, and increased metastatic potential. ${ }^{[1]}$

Thus, inhibition of EGFR signaling represents a novel and rational approach in SCCHN therapeutics. Anti-EGFR monoclonal antibodies (MAbs) have attracted attention as potential candidates in the treatment of SCCHN. Anti-EGFR mAbs, cetuximab and nimotuzumab, are approved molecules and have shown promising results with the improvement in progression-free survival and OS in patients with SCCHN. ${ }^{[12-22]}$

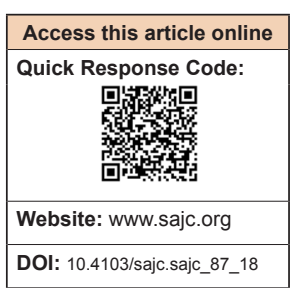

Cetuximab is a chimeric human/murine anti-EGFR mAb. In 2008, the Phase III EXTREME trial demonstrated that the addition of cetuximab to first-line platinum/5-FU chemotherapy significantly leads to a prolonged median progression-free survival of 2.3 months and improved median survival from 7.4 to 10.1 months $(P=0.03)$ compared with platinum-based chemotherapy alone in recurrent and/or metastatic SCCHN. ${ }^{[17]}$ This was the first combination therapy to show a survival benefit over platinum-based chemotherapy in recurrent and/or metastatic SCCHN and has been accepted as standard of care. Overall, cetuximab is associated with a favorable toxicity profile, but safety concerns are raised due to immunogenicity, severe skin toxicity, electrolyte imbalance, infusion reactions, and gastrointestinal adverse events (AEs)..$^{[16,23,24]}$

Nimotuzumab (BIOMAb EGFR ${ }^{\circledR}$ ) is a humanized anti-EGFR $\mathrm{mAb}$. The BEST trial demonstrated that the addition of nimotuzumab to chemo-RT (CRT) or RT provided with long-term survival benefit in inoperable, locally advanced SCCHN. ${ }^{[18]}$

At present, the clinical efficacy and safety of nimotuzumab in LA-SCCHN are well established, and several authors have documented that the addition of the nimotuzumab to various standard treatments (RT or chemotherapy or CRT) improved the tumor response rate and survival with minimal toxicities in LA-SCCHN. ${ }^{[18,20,25-29]}$

However, in recurrent and metastatic SCCHN setting, the clinical evidence on efficacy and survival benefits achieved by the addition of nimotuzumab to standard treatment is not available and needs to be explored. Therefore, in the present study, we retrospectively analyzed the effectiveness, survival benefits, and tolerability of nimotuzumab in combination with

This is an open access journal, and articles are distributed under the terms of the Creative Commons Attribution-NonCommercial-ShareAlike 4.0 License, which allows others to remix, tweak, and build upon the work non-commercially, as long as appropriate credit is given and the new creations are licensed under the identical terms.

For reprints contact: reprints@medknow.com

How to cite this article: Subramanian S, Sridharan N, Balasundaram V, Chaudhari S. Efficacy and safety of nimotuzumab in unresectable, recurrent, and/or metastatic squamous cell carcinoma of the head and neck: A hospital-based retrospective evidence. South Asian J Cancer 2018;7:188-92.
Onepical Oncology and 'Radiation Tamil Nadu, ${ }^{2}$ Medical affairs, Biocon Ltd, Bengaluru, Karnataka, India

Correspondence to: Dr. Nithya Sridharan, E-mail: nithya.hhk@gmail.com 
investigator's choice of standard/conventional treatment in Indian patients with recurrent and/or metastatic SCCHN.

\section{Subjects and Methods}

This retrospective study evaluated the effectiveness and tolerability of nimotuzumab with standard treatment. The hospital records of recurrent/metastatic SCCHN patients who received nimotuzumab in combination with standard/conventional treatment from December 2010 to December 2016 at VS Hospital and Cancer Center, India, were reviewed. The study was approved by the Institutional Review Board.

We selected cases based on the following eligibility criteria: (a) patients aged 18 years and above, (b) patients with recurrent and/ or metastatic carcinoma of the head and neck, (c) histologically confirmed squamous cell carcinoma (d) patients with the Eastern Cooperative Oncology Group (ECOG) performance score $\leq 2$, and (e) patients treated with nimotuzumab (200 mg weekly) combined with standard treatment. We excluded patients of nonhead and neck cancer (HNC), salivary gland cancer, paranasal sinus cancer, and nasopharyngeal cancer and treated before any other anti-EGFR-based therapy. The information was collected from the hospital records of individual patients. Clinical data of patients were collected, including diagnosis, age, gender, pathological type, anatomical subsites, tumor stage, tumor extent, ECOG status, and clinical course of the disease with details of standard treatment and nimotuzumab therapy.

\section{Evaluating parameters}

OS was calculated from the date of diagnosis to the date of death or last contact (visit and telephone). Tumor response was calculated as per the Response Evaluation Criteria in Solid Tumors (RECIST 1.1). Complete remission (CR), partial remission (PR), stable disease, progressive disease (PD), and objective response rate (ORR) were calculated. All AEs were collected and graded by the National Cancer Institute's Common Toxicity Criteria version 4.

\section{Statistical analysis}

Statistical analysis was performed using STATA software (version. 12, StataCorp., College Station, TX: USA). Data were expressed in descriptive statistics. Median OS along with $95 \%$ confidence interval (CI) was estimated by the Kaplan-Meier method.

\section{Results}

The hospital records of 14 patients diagnosed with recurrent and/or metastatic $\mathrm{HNC}$ with histologically confirmed squamous cell carcinoma and treated with nimotuzumab from December 2010 to December 2016 were retrospectively identified. The mean age of the enrolled patients was $57.71 \pm 14.6$ years, with 12 males (86\%) and 2 females (14\%). The most common anatomical site of the tumor was oral cavity $(35.7 \%)$, followed by oropharynx (28.6\%) and hypopharynx (21.4\%). Majority of the patients had a good ECOG performance status $(0-1)$. The baseline characteristics and type of standard combination treatment in recurrent and metastatic SCCHN patients are listed in Table 1.

\section{Effectiveness}

\section{Survival outcome}

In this retrospective study, we observed that OS at 1,2, and 3 years was $77.80 \%$ (95\% CI: 36.48-93.93), 64.81\% (95\% CI: 25.32-87.21), and 64.81\% (95\% CI: 25.32-87.21), respectively. At a median follow-up of 15.17 months, median OS was not reached [Figure 1].

South Asian Journal of Cancer • Volume $7 \bullet$ Issue $3 \bullet$ July-September 2018

\section{Tumor response}

At 24 weeks after completion of treatment, the tumor response rate observed was as follows: $16.7 \%$ of patients had a complete response (CR), 58.3\% of patients had partial response (PR), and $25 \%$ of patients had progressive disease (PD) response. ORR was $75 \%$ [Table 2].

\section{Safety and toxicity}

The most common AEs encountered during nimotuzumab treatment were mucositis $(27.7 \%)$, followed by vomiting $(22.2 \%)$. All AEs were either Grade I (66.7\%) or Grade II (33.3\%). The detailed toxicity and their grades are summarized in Table 3. No Grade III, IV, and V toxicities were observed. Nimotuzumab was observed to be safe with no added toxicity reported.

\section{Discussion}

The findings of the present retrospective study indicate that the addition of nimotuzumab to investigator's choice of standard

Table 1: Baseline characteristics of patients with unresectable recurrent and metastatic squamous cell carcinoma of the head and neck

\begin{tabular}{|c|c|}
\hline Characteristics & Value $(\%)$ \\
\hline Total patients $(n)$ & 14 \\
\hline Mean age (years) \pm SD & $57.71 \pm 14.6$ \\
\hline Median age in years (IQR) & $58.50(46.75,58.50,62.50)$ \\
\hline \multicolumn{2}{|l|}{ Age group (years) } \\
\hline$<65$ & $12(86)$ \\
\hline$\geq 65$ & $2(14)$ \\
\hline \multicolumn{2}{|l|}{ Gender } \\
\hline Male & $12(86)$ \\
\hline Female & $2(14)$ \\
\hline \multicolumn{2}{|l|}{ Performance status } \\
\hline ECOG - 0 & $2(14.3)$ \\
\hline ECOG - 1 & $10(71.4)$ \\
\hline ECOG - 2 & $2(14.3)$ \\
\hline \multicolumn{2}{|l|}{ Anatomical subsites } \\
\hline Hypopharynx & $3(21.4)$ \\
\hline Oral cavity & $5(35.7)$ \\
\hline Oropharynx & $4(28.6)$ \\
\hline Unknown primary & $2(14.3)$ \\
\hline \multicolumn{2}{|l|}{ Extent of disease } \\
\hline Only locoregionally recurrent & $3(21.4)$ \\
\hline $\begin{array}{l}\text { Metastatic with or without } \\
\text { locoregional recurrence }\end{array}$ & $11(78.6)$ \\
\hline \multicolumn{2}{|l|}{ Type of treatment regimen } \\
\hline $\mathrm{CT}+$ nimotuzumab & $1(7.1)$ \\
\hline CTRT + nimotuzumab & $8(57.1)$ \\
\hline $\begin{array}{l}\text { ICT + nimotuzumab, CTRT } \\
+ \text { nimotuzumab }\end{array}$ & $1(7.1)$ \\
\hline ICT, CTRT + nimotuzumab & $4(28.7)$ \\
\hline
\end{tabular}

$\mathrm{SD}=$ Standard deviation, $\mathrm{IQR}=$ Interquartile range, $\mathrm{ECOG}=$ Eastern Cooperative Oncology Group, CT=Chemotherapy, CTRT=Chemoradiotherapy, ICT=Induction chemotherapy

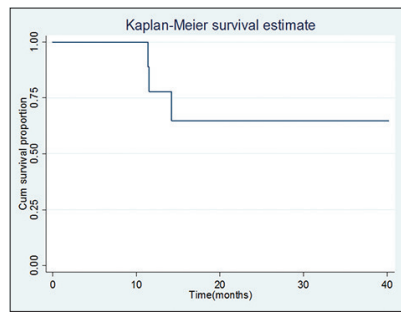

Figure 1: Kaplan-Meier method estimates of overall survival in recurrent and metastatic squamous cell carcinoma of the head and neck on treatment 
Table 2: Overall tumor response in patients treated with nimotuzumab and standard treatment $(n=12)$

\begin{tabular}{|c|c|c|c|}
\hline \multicolumn{3}{|c|}{ Overall tumor response } & $n^{*}(\%)$ \\
\hline \multicolumn{3}{|l|}{$\mathrm{CR}$} & $2(16.7)$ \\
\hline \multicolumn{3}{|l|}{ PR } & $7(58.3)$ \\
\hline \multicolumn{3}{|l|}{ SD } & 0 \\
\hline \multicolumn{3}{|l|}{ PD } & $3(25)$ \\
\hline \multicolumn{3}{|l|}{ ORR } & $9(75)$ \\
\hline \multicolumn{3}{|c|}{$\begin{array}{l}\text { Table 3: Common adverse events encountered during } \\
\text { the treatment }\end{array}$} & $\begin{array}{l}\mathrm{R}=\text { Partial } \\
\text { response rate } \\
\text { uring }\end{array}$ \\
\hline Adverse events & Grade $1(\%)$ & Grade $2(\%)$ & Total $(\%)$ \\
\hline Mucositis & $3(16.8)$ & $2(11.1)$ & $5(27.7)$ \\
\hline Vomiting & $2(11.1)$ & $2(11.1)$ & $4(22.2)$ \\
\hline Neutropenia & $2(11.1)$ & 0 & $2(11.1)$ \\
\hline Anemia & $1(5.5)$ & $1(5.5)$ & $2(11.1)$ \\
\hline Diarrhea & $1(5.5)$ & 0 & $1(5.5)$ \\
\hline Rash & $2(11.1)$ & $1(5.5)$ & $3(16.8)$ \\
\hline Fatigue & $1(5.5)$ & 0 & $1(5.5)$ \\
\hline Total & $12(66.7)$ & $6(33.3)$ & $18(100)$ \\
\hline
\end{tabular}

treatment raised the tail of the survival curve, which signifies an improved survival rate in unresectable, recurrent and/or metastatic SCCHN.

Recurrent and metastatic SCCHN is a rising problem in India and a clinical challenge to manage despite the treatment options and strategies currently available. ${ }^{[4]}$ Recurrent and metastatic SCCHN carries a poor prognosis with a median OS less than a year. Most patients with R/M SCCHN qualify either for palliative chemotherapy or best supportive care as surgery and RT are generally not an option. ${ }^{[10]}$

Palliative systemic chemotherapy with or without radiation therapy remains the mainstay of treatment in recurrent/metastatic, unresectable with the disease. The choice of chemotherapy depends on patient characteristics, side-effect profile, and patient performance status. Platinum-based chemotherapy and platinum/5-FU/taxane-based regimes are currently the standard of care in the management of SCCHN that is recurrent, metastatic, unresectable, and considered incurable. ${ }^{[4,8-10]}$ Unfortunately, the survival outcome is dismal, and efforts to improve the outcomes have led to molecular biology-based targeted therapy.

EGFR represents a novel molecular target in HNCs. The overexpression of the EGFR levels is closely related to tumor cell growth, proliferation, invasion, metastasis, apoptosis, and poor prognosis. Thus, inhibition of EGFR leads to inhibition of tumor cell proliferation, differentiation, and tumor angiogenesis and also improves the treatment response of chemotherapy and radiation. ${ }^{[30]}$

The EXTREME trial demonstrated that the addition of the EGFR-targeted monoclonal antibody, cetuximab to first-line platinum, and 5-FU chemotherapy significantly leads to a prolonged median progression-free survival of 2.3 months (hazard ratio [HR] for progression, 0.54; $P<0.001$ ), improved OS by 2.7 months (10.1 vs. 7.4 months for platinum-based chemotherapy alone), and improved the chance of a response compared with platinum-based chemotherapy alone in recurrent and/or metastatic SCCHN. ${ }^{[16,17]}$

In general, cetuximab has been accepted as standard of care in recurrent and/or metastatic SCCHN, but safety concerns are raised 190 due to immunogenicity, severe skin toxicity, electrolyte imbalance, infusion reactions, and gastrointestinal AEs. ${ }^{[16,23,24]}$ In the clinical trial by Vermorken et al., comparing chemotherapy versus chemotherapy plus cetuximab, there was $20 \%$ discontinuation rate due to toxicities in the chemotherapy plus cetuximab group with nine cases of sepsis. ${ }^{[16]}$ Similarly, other anti-EGFR mAbs also have potential risk and safety concerns. ${ }^{[16,23,24]}$

Nimotuzumab (BIOMAb EGFR ${ }^{\circledR}$ ) is a humanized anti-EGFR $\mathrm{mAb}$ that binds to the extracellular domain of EGFR with intermediate affinity and high specificity which results in the blockade of receptor-dependent signal transduction pathways and provides antitumor efficacy. ${ }^{[18,31]}$ It also enhances the tumor radiosensitivity by inhibiting the radiation-induced activation of DNA-PKcs by blocking the PI3K/AKT pathway. ${ }^{[32]}$

Nimotuzumab unique benign low toxicity profile can be attributed to the fact that unlike other anti-EGFR antibodies, it requires bivalent binding for stable attachment, leading to selective binding to tumors cells that overexpress the EGFR (moderate to high) level. When EGFR expression is low, such as in normal tissues, monovalent interaction of nimotuzumab is transient, thus sparing normal healthy tissues and avoiding severe toxicities. ${ }^{[33]}$ This probably explains the minimal toxicities associated with nimotuzumab therapy.

The promising therapeutic and survival outcomes documented in the BEST trial strongly favored and laid the foundation for nimotuzumab as an add-on therapy to standard treatments in LA-SCCHN. ${ }^{[18]}$ The BEST trial was a multicenter, randomized, open-label, Phase IIb, 5-year study and the first of its kind cohort conducted among the Indian population. ${ }^{[11]}$ The study evaluated the safety and efficacy of nimotuzumab, administered along with CRT or RT in patients with inoperable, locally advanced SCCHN. The trial documented that the 5-year OS was significantly higher in the nimotuzumab + CRT group than in the CRT group $(57 \%$ vs. $26 \%, P=0.03)$. The median 5 -year OS was not reached in the nimotuzumab + CRT group at 60 months, whereas it was 21.94 months in the CRT group $(P=0.0078)$; the addition of nimotuzumab to CRT caused a $64 \%$ reduction in death risk $(\mathrm{HR}=0.36,95 \% \mathrm{CI}: 0.37,1.56)$. The 5 -year OS was $39 \%$ (95\% CI: 19.71, 61.46) in the nimotuzumab + RT group versus $26 \%(95 \% \mathrm{CI}: 10.23,48.41 ; P>0.05)$ in the RT group. Nimotuzumab was found to be safe and well tolerated with few mild-to-moderate self-limiting AEs. No long-term drug-related toxicity was seen during the median follow-up of 65.7 months. Results of the BEST trial strongly suggested that nimotuzumab improved locoregional control and prolonged survival when used along with RT or CRT. ${ }^{[18]}$

In India, several authors in their individual research have documented that the addition of nimotuzumab to various standard treatment/combined treatment strategies (RT or chemotherapy or concurrent chemoradiation) in LA-SCCHN improved tumor response rate and survival outcomes with minimal toxicities. ${ }^{[19,20,25-29]}$ However, its role in recurrent and metastatic SCCHN setting is not well explored.

To the best of our knowledge, there is no published literature of Indian patients regarding the use of nimotuzumab in recurrent and metastatic SCCHN. This is the first kind of retrospective study, which provides evidence on the effectiveness, survival benefits, and tolerability of nimotuzumab with investigator's South Asian Journal of Cancer $\bullet$ Volume 7 Issue 3 July-September 2018 
choice of standard care therapy/conventional treatment in Indian patients with recurrent and metastatic SCCHN.

In the present retrospective study, we found that addition of nimotuzumab to the standard treatment resulted in improved survival rates in unresectable, recurrent, and metastatic SCCHN. The OS benefit achieved with the add-on combination at 1,2 , and 3 years was $77.80 \%, 64.81 \%$, and $64.81 \%$, respectively. At a median follow-up of 15.17 months, median OS was not reached.

Our findings of improved survival benefit in recurrent and metastatic SCCHN are similar to survival benefit observed in LA-SCCHN setting. ${ }^{[18,20,25-30]}$ The improved OS achieved with the addition of nimotuzumab to the standard treatment in the present analysis is higher than the documented landmark EXTREME cetuximab study. ${ }^{[17]}$ Thus, nimotuzumab as an add-on to standard treatment can be a promising option in recurrent and metastatic SCCHN.

In the present study, the common AEs observed during treatment were mucositis, followed by vomiting, which are similar to documented studies with nimotuzumab. ${ }^{[11,14-20]}$ All AEs were either Grade I or Grade II. No Grade III, IV, and V AEs were observed. No typical anti-EGFR-related toxicity such as severe skin rash, infusion reactions, or hypomagnesemia was observed. Nimotuzumab was observed to be safe with no additional potentiating AEs encountered.

The study had the following limitations; first, the study design was retrospective with a single-arm assessment. Second, the analyzed retrospective data included heterogeneous standard treatment regimens, and third, the sample size was small.

\section{Conclusions}

In the first of its kind study, the addition of nimotuzumab to standard treatment showed promising survival outcomes as well as the response rate in recurrent/metastatic SCCHN patients without producing additional toxicity. Robust multicenter, randomized trials are warranted to validate these interesting findings.

\section{Acknowledgment}

We would like to thank Ramez Ahmed, for his valuable inputs and transcribing the manuscript.

\section{Financial support and sponsorship}

Nil.

\section{Conflicts of interest}

There are no conflicts of interest.

\section{References}

1. Jemal A, Bray F, Center MM, Ferlay J, Ward E, Forman D, et al. Global cancer statistics. CA Cancer J Clin 2011;61:69-90.

2. Graham S, Dayal H, Rohrer T, Swanson M, Sultz H, Shedd D, et al. Dentition, diet, tobacco, and alcohol in the epidemiology of oral cancer. J Natl Cancer Inst 1977;59:1611-8.

3. Joshi P, Dutta S, Chaturvedi P, Nair S. Head and neck cancers in developing countries. Rambam Maimonides Med J 2014;5:e0009.

4. Parikh P, Patil V, Agarwal JP, Chaturvedi P, Vaidya A, Rathod S, et al. Guidelines for treatment of recurrent or metastatic head and neck cancer. Indian J Cancer 2014;51:89-94.

5. Dayal PK, Mani NJ, Bhargava K. Prevalence of oral cancer and precancerous lesions in 'pan' /'supari' chewers. Indian J Public Health 1978;22:234-45.

6. Jacob LA, Chaudhuri T, Lakshmaiah KC, Babu KG, Dasappa L, Babu M, et al. Current status of systemic therapy for recurrent and/or metastatic squamous cell carcinoma of the head and neck. Indian J Cancer 2016;53:471-7.

7. Tuljapurkar V, Dhar H, Mishra A, Chakraborti S, Chaturvedi P, Pai PS, et al. The Indian scenario of head and neck oncology - Challenging the dogmas. South Asian J Cancer 2016;5:105-10.

South Asian Journal of Cancer Volume 7 Issue 3 July-September 2018
8. Marur S, Forastiere AA. Update on role of chemotherapy in head and neck squamous cell cancer. Indian J Surg Oncol 2010;1:85-95.

9. Molin Y, Fayette J. Current chemotherapies for recurrent/metastatic head and neck cancer. Anticancer Drugs 2011;22:621-5.

10. Vermorken JB, Specenier P. Optimal treatment for recurrent/metastatic head and neck cancer. Ann Oncol 2010;21 Suppl 7:vii252-61.

11. Agulnik M. New approaches to EGFR inhibition for locally advanced or metastatic squamous cell carcinoma of the head and neck (SCCHN). Med Oncol 2012;29:2481-91.

12. Vermorken JB, Trigo J, Hitt R, Koralewski P, Diaz-Rubio E, Rolland F, et al. Open-label, uncontrolled, multicenter phase II study to evaluate the efficacy and toxicity of cetuximab as a single agent in patients with recurrent and/or metastatic squamous cell carcinoma of the head and neck who failed to respond to platinum-based therapy. J Clin Oncol 2007;25:2171-7.

13. Baselga J, Trigo JM, Bourhis J, Tortochaux J, Cortés-Funes H, Hitt R, et al. Phase II multicenter study of the antiepidermal growth factor receptor monoclonal antibody cetuximab in combination with platinum-based chemotherapy in patients with platinum-refractory metastatic and/or recurrent squamous cell carcinoma of the head and neck. J Clin Oncol 2005;23:5568-77.

14. Herbst RS, Arquette M, Shin DM, Dicke K, Vokes EE, Azarnia N, et al. Phase II multicenter study of the epidermal growth factor receptor antibody cetuximab and cisplatin for recurrent and refractory squamous cell carcinoma of the head and neck. J Clin Oncol 2005;23:5578-87.

15. Burtness B, Goldwasser MA, Flood W, Mattar B, Forastiere AA; Eastern Cooperative Oncology Group. et al. Phase III randomized trial of cisplatin plus placebo compared with cisplatin plus cetuximab in metastatic/recurrent head and neck cancer: An eastern cooperative oncology group study. J Clin Oncol 2005;23:8646-54.

16. Vermorken JB, Mesia R, Rivera F, Remenar E, Kawecki A, Rottey S, et al. Platinum-based chemotherapy plus cetuximab in head and neck cancer. N Engl J Med 2008;359:1116-27.

17. Rivera F, García-Castaño A, Vega N, Vega-Villegas ME, Gutiérrez-Sanz L. Cetuximab in metastatic or recurrent head and neck cancer: The EXTREME trial. Expert Rev Anticancer Ther 2009;9:1421-8.

18. Reddy BK, Lokesh V, Vidyasagar MS, Shenoy K, Babu KG, Shenoy A, et al. Nimotuzumab provides survival benefit to patients with inoperable advanced squamous cell carcinoma of the head and neck: A randomized, open-label, phase Ilb, 5-year study in Indian patients. Oral Oncol 2014;50:498-505.

19. Babu KG, Viswanath L, Reddy BK, Shenoy K, Shenoy A, Naveen T. et al. An open-label, randomized, study of h-R3mAb (nimotuzumab) in patients with advanced (stage III or IVa) squamous cell carcinoma of head and neck (SCCHN): Four-year survival results from a phase Ilb study. J Clin Oncol 2010;28 Suppl 15:428s.

20. Subramanium S, Balasundaram V, Nithya S, Kiran P. Nimotuzumab with induction chemotherapy and chemo-radiation in patients with advanced head and neck cancer. J Cancer Ther 2015;6: 146-52.

21. Crombet T, Osorio M, Cruz T, Roca C, del Castillo R, Mon R, et al. Use of the humanized anti-epidermal growth factor receptor monoclonal antibody h-R3 in combination with radiotherapy in the treatment of locally advanced head and neck cancer patients. J Clin Oncol 2004;22: 1646-54.

22. Rodríguez MO, Rivero TC, del Castillo Bahi R, Muchuli CR, Bilbao MA, Vinageras EN, et al. Nimotuzumab plus radiotherapy for unresectable squamous-cell carcinoma of the head and neck. Cancer Biol Ther 2010;9:343-9.

23. Guan M, Zhou YP, Sun JL, Chen SC. Adverse events of monoclonal antibodies used for cancer therapy. Biomed Res Int 2015;2015:428169.

24. Li X, Shan BE, Wang J, Xing LP, Guo XJ, Zhang YH, et al. Incidence and risk of treatment-related mortality with anti-epidermal growth factor receptor monoclonal antibody in cancer patients: A meta-analysis of 21 randomized controlled trials. PLoS One 2013;8:e81897.

25. Bhatnagar AR, Singh DP. A comparative study of a monoclonal antibody against EGFR (nimotuzumab) used in combination with chemoradiation versus chemoradiation alone in the treatment of locally advanced inoperable squamous cell carcinoma of the head and neck. J Clin Oncol 2012;30 Suppl 30:51.

26. Kumar A, Mishra KC. To assess the possibility of combining MAb (nimotuzumab) with concurrent chemoradiation in patients with locally advanced squamous cell carcinoma of head and neck. Scientific abstracts. Indian J Med Paediatr Oncol 2012;33:1.

27. Somani N. Nimotuzumab with concurrent chemo-radiotherapy in patients with locally advanced squamous cell carcinoma of head and neck. J Cancer Ther 2015;6:356-61. 
28. Kondaveeti SS, Sundaram R. Therapeutic effect of concurrent radiation with single agent nimotuzumab in locally advanced and recurrent HNSCC: Retrospective analysis. Int J Radiat Oncol 2014;90:S561.

29. Talapatra K, Deshpande M, Goyle S, Majumder D, Badhwar S, Mistry R. Nimotuzumab and intensity modulated radiation therapy in the concurrent setting of locally advanced head and neck cancers: Early results of a prospective trial in India. Int J Radiat Oncol 2016;96:E359.

30. Wykosky J, Fenton T, Furnari F, Cavenee WK. Therapeutic targeting of epidermal growth factor receptor in human cancer: Successes and limitations. Chin J Cancer 2011;30:5-12.
31. Perez R, Moreno E, Garrido G, Crombet T. EGFR-targeting as a biological therapy: Understanding nimotuzumab's clinical effects. Cancers (Basel) 2011;3:2014-31.

32. Qu YY, Hu SL, Xu XY, Wang RZ, Yu HY, Xu JY, et al. Nimotuzumab enhances the radiosensitivity of cancer cells in vitro by inhibiting radiation-induced DNA damage repair. PLoS One 2013;8:e70727.

33. Garrido G, Tikhomirov IA, Rabasa A, Yang E, Gracia E, Iznaga N, et al. Bivalent binding by intermediate affinity of nimotuzumab: A contribution to explain antibody clinical profile. Cancer Biol Ther 2011;11:373-82. 